\title{
Utopies sanitaires, entre fitness et découverte de soi-même
}

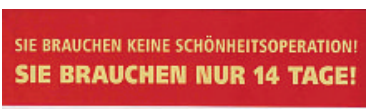

DER ULTIMATIVE

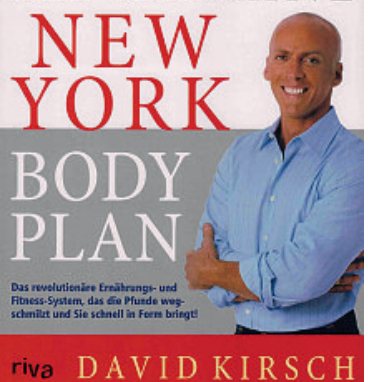

Une marée de guides inonde le marché du livre. Leur point commun: une approche selon laquelle la santé s'obtient par un travail personnel.
1 Le lecteur francophone intéressé trouvera des guides de santé populaires sur le site internet www.payot.ch $\rightarrow$ Santé, diététique, sport $\rightarrow$ meilleures ventes.

2 Vous trouverez la littérature sur Internet sous www.bullmed.ch $\rightarrow$ archives $2010 \rightarrow 16$.

* PD Dr rer. soc. Eberhard Wolff est licencié en science culturelles, historien de la Médecine et membre de la rédaction Histoire de la médecine du Bulletin des médecins suisses.
Qu'entend-on par santé? Pitié! Evitons ici les thèses théoriques! La définition utopique de l'OMS selon laquelle la santé est un état de complet bien-être physique, mental et social nous a été servie bien assez souvent.

Toutefois, récemment, j'ai fait une nouvelle découverte qui m'a permis un aperçu plus précis de ce que les Suisses alémaniques ${ }^{1}$ entendent par santé. J'avais à établir une synthèse des guides de santé les plus appréciés de ces dernières années. Les données sérieuses collectées par Media Control pour les éditeurs et libraires suisses alémaniques sont publiées sur www.hitparade.ch, c'est-à-dire juste à côté de Martin Suter et Lady Gaga.

Le hit-parade des livres constitue l'épicentre suisse d'un genre littéraire étonnamment hétérogène. Dans le peloton de tête, on ne trouve qu'un seul livre «de médecine de famille» traditionnel, celui de Grönemeyer (mais oui, il s'agit bien du frère de l'artiste, du nouveau médecin star des médecins alternatives) $[1]^{2}$. Le genre s'est multiplié à la manière d'un caléidoscope. Les guides de santé se classent facilement dans les ouvrages de sport ou de cuisine. Ils se déguisent en journaux intimes ou récits. Le très célèbre «Babyjahre» de Remo Largo [2] n'est pas non plus, à proprement parler, un mode d'emploi.

Mais quel est le rapport entre ces guides populaires et l'image utopique d'un état de complet bien-être physique, mental et social? Il est peut-être plus important qu'on ne l'imagine! Naturellement, ces ouvrages sont centrés sur le bien-être physique des lecteurs, mais lequel? Il s'agit parfois de certaines maladies aiguës, par exemple le burn out [3]. Mais nombre de ces bestsellers n'évoquent pas un corps affaibli, mais bien un corps sain: la manière de conserver la «forme» (dans «Der ultimative New York Body Plan» [4]), la manière de lui apporter de la «vitalité» (avec la méthode «Body Reset» [5]), et celle de le garder «forever young» (toujours jeune) (cf. le guide «Metabolic Power» [6]). Tous ces livres promettent un corps «svelte», même en dormant [7], en forme, plein de vitalité et jeune: s'il ne s'agit pas là d'une utopie de la santé physique, et même d'un physique de réussite et de performance!

Et qu'en est-il du bien-être social selon l'OMS? On y pense beaucoup également. «Body Reset» promet que l'on peut devenir attirant pour la vie grâce à cette méthode. Ceci n'est rien d'autre qu'un facteur de bienêtre social. Cela vaut même pour les guides médicaux, par exemple le journal intime de Raaflaub sur l'impuissance «Tote Hose» (Pantalon mort), dont le sous-titre précise: «Worüber Männer schweigen» (ce dont les hommes ne parlent pas) [8]. La plupart des guides considèrent la santé et la maladie comme un processus social et de communication, ce que pensent aussi les lecteurs.

Certains best-sellers ont pour but d'aider les gens à accéder à une relation satisfaisante avec eux-mêmes, par exemple le guide anti-minceur «Das Ich-Gewicht» (le poids qui m'est propre) de Maja Storch [9]. L'ouvrage «Wendepunkte im Lebenslauf» (changements de vies) du psychothérapeute Jürg Willi insiste sur la nécessité d'harmoniser la vie personnelle et publique dans un monde changeant [10]. Sans parler des instructions sur l'art de vivre («Lebenskunst») [11] de Grönemeyer. Ceci n'est pas différent de l'objectif de bien-être social

De tels livres se focalisent donc aussi sur le bienêtre mental, par exemple lorsqu'il s'agit de se trouver soi-même. «Das heilende Bewusstsein» (la conscience de soi qui guérit) de Joachim Faulstich va encore plus loin, s'interrogeant sur la capacité curative de l'esprit [12].

Quelle que soit l'attitude adoptée face aux messages de ces livres, on ne peut leur reprocher de vision trop restrictive. Au contraire: peut-être que la grande utopie sanitaire de l'OMS s'est mieux développée ici que dans des lieux plus pragmatiques, même si, dans les détails, on ne peut vraiment la comprendre dans le sens de l'OMS.

Le genre reflète en revanche des utopies de santé plus larges qui dépassent la définition de l'OMS: la santé vue comme quelque chose que l'on peut acquérir soi-même, que ce soit en mangeant, en bougeant ou en travaillant sur soi-même; la santé comme pratique et non comme état, tout à fait au sens de notre société de bien-être, ou la santé comme quelque chose de très personnel, propre à chaque individu et non comme un ensemble encyclopédique parfois un peu hermétique, de manière à prendre en compte l'intérêt particulier de chacun, sa propre utopie de bien-être.

Notre conception de la santé fait l'objet d'un vote. Pas seulement dans les urnes, mais également dans les librairies, le porte-monnaie faisant office de bulletin de vote.

Eberhard Wolff* 


\section{Références}

1 Grönemeyer D. Grönemeyers neues Hausbuch der Gesundheit. Reinbek: Rowohlt; 2008.

2 Largo R. Babyjahre. Die frühkindliche Entwicklung aus biologischer Sicht. München: Piper; 2009.

3 Knapp T, Baer N. In den Krallen des Raubvogels. Der Burn-out Erfahrungsbericht. Olten: Textwerkstatt; 2008.

4 Kirsch D. Der ultimative New York Body Plan. München: Riva; 2007.

5 Gehring J. Mit Body Reset attraktiv, schlank, vital, ein Leben lang. Kreuzlingen: Sivita; 2009.

6 Strunz U. Die neue Diät. Fit und schlank durch Metabolic Power. München: Heyne; 2008.

7 Pape D et al. Schlank im Schlaf. München: Gräfe \& Unzer; 2006.
8 Raaflaub W. Tote Hose. Worüber Männer schweigen. Gockhausen: Wörterseh; 2007.

9 Storch M. Mein Ich-Gewicht. Wie das Unbewusste hilft, das richtige Gewicht zu finden. München, Zürich: Pendo; 2008.

10 Willi J. Wendepunkte im Lebenslauf. Persönliche Entwicklung unter veränderten Umständen die ökologische Sicht der Psychotherapie. Stuttgart: Klett-Cotta; 2007.

11 Grönemeyer D. Lebe mit Herz und Seele. Sieben Haltungen zur Lebenskunst. Freiburg/Br.: Herder; 2009.

12 Faulstich J. Das heilende Bewusstsein. Wunder und Hoffnung an den Grenzen der Medizin. München: Knaur; 2006. 